\title{
Chapter 4. Lifestyle modifications
}

\author{
Hypertension Research (2014) 37, 286-290; doi:10.1038/hr.2014.7
}

\section{POINT 4}

1. Lifestyle modifications are important for ameliorating hypertension, both before and after the start of antihypertensive drug therapy. (Recommendation grade: A, Consensus)

2. Salt reduction: The target of salt reduction is $<6 \mathrm{~g}$ per day. (Recommendation grade: A, Evidence level: I)

3. Dietary pattern: Fruit/vegetable intake should be increased, and cholesterol/saturated fatty acid intake should be reduced. Fish (fish oil) intake should also be increased. (Recommendation grade: B, Evidence level: II)

4. Weight control: The target body mass index ([body weight $(\mathrm{kg})] \div$ [height $\left.(\mathrm{m})]^{2}\right)$ is $<25 \mathrm{~kg} \mathrm{~m}^{-2}$. Even when the target is not reached, a significant decrease in blood pressure can be achieved by reducing body weight by $\sim 4 \mathrm{~kg}$. (Recommendation grade: A, Evidence level: I)

5. Exercise: Primarily periodic ( $30 \mathrm{~min}$ or longer, daily, if possible) and aerobic exercise should be practiced. (Recommendation grade: A, Evidence level: I)

6. Reduction of alcohol intake: Alcohol intake should be restricted. (Recommendation grade: A, Evidence level: I)

7. Quitting smoking: Smoking cessation should be promoted, and passive smoking must be avoided. (Recommendation grade: A, Consensus, Evidence level: IVa)

8. Others: Exposure to cold should be avoided. Emotional stress should be managed. (Recommendation grade: $\mathrm{C} 1$, Evidence level: IVa)

9. Comprehensive lifestyle modifications are more effective. (Recommendation grade: B, Evidence level: II)

Lifestyle modifications can lead to a mild decrease in blood pressure, increasing the actions of antihypertensive drugs, thus contributing to a reduction in the dose. Therefore, lifestyle modifications should be actively performed not only before but also after the start of antihypertensive drug therapy. All hypertensive patients should receive education and guidance regarding lifestyle modifications to prevent cardiovascular disease and ameliorate risk factors other than hypertension. In addition, from the perspective of hypertension prevention, healthy people should also practice appropriate lifestyle behavior. In particular, lifestyle behavior is established during childhood, and a disturbed lifestyle during young age can cause physical abnormalities over one's lifetime; therefore, it is important to provide guidance/education to children on adequate lifestyle behavior. Table 4-1 lists the points of lifestyle modification, and Figure 4-1 shows the expected decrease in blood pressure related to respective lifestyle modifications.

\section{SALT REDUCTION}

Observational studies including the INTERSALT ${ }^{51}$ have indicated the association between excessive salt intake and an increase in blood pressure. In addition, many interventional studies in Western countries, such as the DASH-Sodium, ${ }^{339}$ have also shown the hypotensive effects of salt reduction. ${ }^{340-342}$ According to the results of these interventional studies, blood pressure was not significantly decreased without reducing salt intake to $<6.5 \mathrm{~g}$ per day. In the TONE study, salt restriction to $<5.6 \mathrm{~g}$ per day was required for maintaining normal blood pressure after the discontinuation of antihypertensive drugs. ${ }^{343}$ On the basis of these results, Western guidelines on the management of hypertension recommend a salt intake of $<6$ g per day. ${ }^{344}$ In the WHO Guidelines recently published (for the general population), a salt intake of $<5 \mathrm{~g}$ per day is strongly recommended. ${ }^{345}$ However, we set a target restricting salt intake to $<6$ g per day, considering actual circumstances in Japan. The Committee for Dietary Salt Reduction of the Japanese Society of Hypertension provides useful recipes as a reference to achieve a daily diet with $<6$ g per day. ${ }^{346}$

It is reported that salt intake was originally $0.5-3 \mathrm{~g}$ per day in humans belonging to the Stone Age. Some investigators have stated that a low salt intake is appropriate for humans as an organism. However, interventional studies have confirmed that salt intake can be safely reduced to $3.8 \mathrm{~g}$ per day on average. ${ }^{339}$ In recent Western guidelines/recommendations on diet, a target or ideal salt intake of $<3.8 \mathrm{~g}$ per day is recommended, ${ }^{344}$ aiming at a lower salt intake. Many epidemiological studies and their meta-analyses ${ }^{347}$ have shown that excessive salt consumption increases the risk for cardiovascular disease. A meta-analysis of four interventional studies involving follow-up for 6 months or more ${ }^{348}$ and a long-term follow-up study following the completion of an interventional study ${ }^{349}$ also showed similar results. In the interventional studies cited in these reports, ${ }^{348,349}$ salt intake was reduced to $\sim 6$ g per day. The inhibitory effects of strict salt reduction on the risk of cardiovascular disease should be examined in the future. On the basis of the results of epidemiological studies, salt restriction tends to potently inhibit the risk for stroke in comparison with that for coronary artery disease. ${ }^{340}$ This may be because the influence of blood pressure on stroke is very marked, whereas factors other than blood pressure also contribute to coronary artery disease.

In Japan, the mean salt intake still exceeds $10 \mathrm{~g}$ per day. ${ }^{53}$ Although the necessity of salt reduction has been emphasized often, a target value of $<6 \mathrm{~g}$ per day is not achieved in many hypertensive patients. However, as the effectiveness of salt reduction is considered to depend on its intensity (a meta-analysis indicated a decrease in systolic blood pressure of about $1 \mathrm{~mm} \mathrm{Hg}$ with a decrease in salt intake of $1 \mathrm{~g}$ per day ${ }^{341}$ ), guidance should be given to gradually reduce salt intake over a 


\section{Table 4-1 Points of lifestyle modifications}

\begin{tabular}{ll}
\hline $\begin{array}{l}\text { 1. Salt reduction } \\
\text { 2a. Vegetables/fruit } \\
\text { 2b. Lipids }\end{array}$ & $\begin{array}{l}<6 \text { g per day } \\
\text { Increased intake of vegetables/fruits }\end{array}$ \\
& $\begin{array}{l}\text { Reduced intake of cholesterol and saturated } \\
\text { fatty acids, increased intake of fish (fish oil) }\end{array}$ \\
3. Weight loss & BMI ([body weight $\left.(\mathrm{kg})] \div[\text { height }(\mathrm{m})]^{2}\right):<25 \mathrm{~kg} \mathrm{~m}^{-2}$ \\
4. Exercise & $\begin{array}{l}\text { In hypertensive patients with no cardiovascular disease, } \\
\text { exercise, primarily aerobic exercise, should be performed } \\
\text { periodically (for } \geqslant 30 \text { min daily, if possible). }\end{array}$ \\
& $\leqslant 20-30 \mathrm{ml}$ per day in men and $\leqslant 10-20 \mathrm{ml}$ \\
5. Reduction of & per day in women as ethanol \\
alcohol intake & including the prevention of passive smoking \\
6. Smoking cessation
\end{tabular}

Combined lifestyle modifications are more effective.

${ }^{a} \mathrm{An}$ increased intake of vegetables/fruits is not recommended for patients with severe renal dysfunction because of the risk of hyperkalemia. An excessive intake of fruit with a high fructose content is not recommended in patients who need to restrict their energy intake, such as obese and diabetic patients.

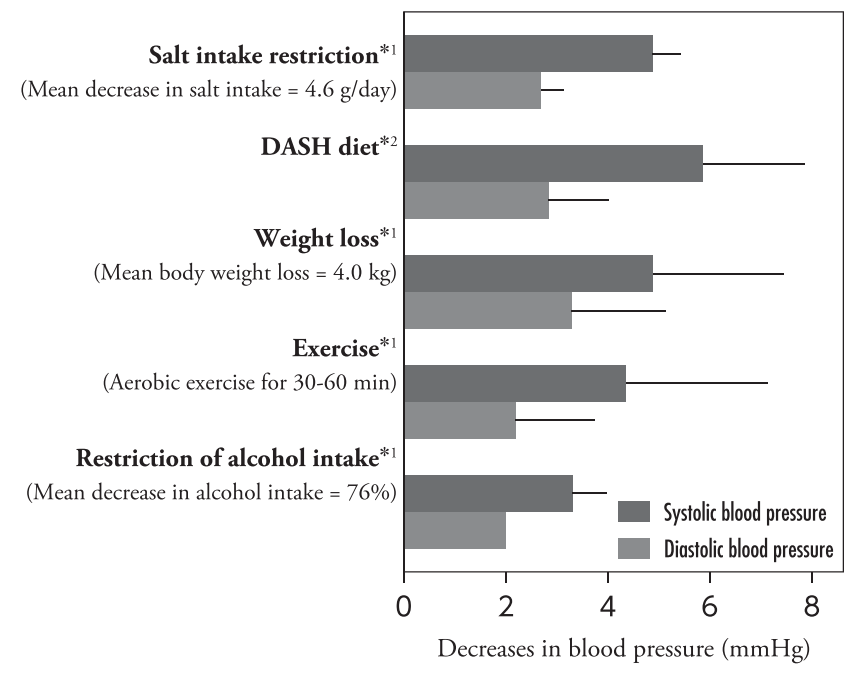

${ }^{*}$ 1. metaanalysis, ${ }^{*} 2$. randomized intervention study

Salt intake restriction [341], DASH diet [339], weight loss [374], exercise [342], restriction of alcohol intake [382]

Figure 4-1 Decreases in blood pressure levels through lifestyle modifications. *1. meta-analysis, *2. randomized intervention study. Salt intake restriction, ${ }^{341}$ DASH diet, ${ }^{339}$ weight loss, ${ }^{374}$ exercise, ${ }^{342}$ restriction of alcohol intake. ${ }^{382}$

long period. Furthermore, very strict salt restriction may lead to dehydration, affecting the physical condition; therefore, salt reduction must be carefully performed in elderly persons and in patients with chronic kidney disease, in whom the Na-retaining capacity of the kidney is decreased, or in summer, when water loss is marked. ${ }^{350}$ As salt intake is greater with higher energy intake, energy restriction can reduce salt intake.

Presently, in Japan, the Na but not the salt content is required to be included in the nutritional information on processed foods, but dietary guidance is given in terms of salt content $(\mathrm{g})$. Therefore, the $\mathrm{Na}$ content must be converted to the salt content. Although the salt content can be calculated by multiplying the $\mathrm{Na}$ content by 2.54 , the $\mathrm{Na}$ content is multiplied by 2.5 for simplification in clinical practice. Salt intake is different among subjects, and the guidance for salt reduction should be given on the basis of individual salt intake. If salt intake is evaluated in advance, better guidance can be given. The assessment of the $\mathrm{Na} /$ creatinine $[\mathrm{Cr}]$ ratio using second urine collected early in the morning or spot urine is practical at general medical facilities (Table 4-2). ${ }^{351}$ The reliability of findings can be improved using a calculation formula incorporating the estimated 24-h urinary $\mathrm{Cr}$ excretion. However, even values calculated using the formula have limitations. Therefore, measurements should be recorded several times, and, simultaneously, an interview on diet may also be conducted.

It is difficult to practice an ideal salt restriction regimen only through the efforts of the patient and his/her caregivers in the present social environment, and political approaches are necessary. In Japan, $90 \%$ of salt intake is ingested from processed foods containing soy sauce or miso, ${ }^{352}$ and strategies to reduce the salt content of processed foods are necessary to achieve the target of salt reduction. Furthermore, salt restriction during childhood may prevent an increase in blood pressure over a long period. ${ }^{353}$ In Japanese children, salt intake is high, ${ }^{354}$ and education/guidance for children and their mothers should be given/improved.

\section{NUTRIENTS AND FOODS}

In the United States, an interventional study of the DASH diet, which is rich in vegetables, fruit and low-fat dairy products (low in saturated fatty acids and cholesterol and high in $\mathrm{Ca}, \mathrm{K}, \mathrm{Mg}$ and dietary fiber), was conducted. ${ }^{288,339}$ This diet was shown to have a significant hypotensive effect. Of the components of the DASH diet, the hypotensive effects of $\mathrm{K}$ are weak, and are mentioned only in some guidelines such as AHA reports on dietary therapy for hypertension. ${ }^{355}$ However, dietary $\mathrm{K}$ markedly counteracts against an increase in blood pressure due to an excessive salt intake. ${ }^{356,357}$ As food processing causes $\mathrm{Na}$ addition and $\mathrm{K}$ loss, the increased consumption of $\mathrm{K}$ should be recommended in industrialized countries where processed foods are commonly used. An epidemiological study reported that the $\mathrm{Na} / \mathrm{K}$ intake ratio was predictive of an increase in the risk for cardiovascular disease or total mortality. ${ }^{358}$ In the WHO Guidelines recently published, ${ }^{359}$ a $\mathrm{K}$ intake of $3510 \mathrm{mg}$ per day or more is recommended. However, an increased intake of vegetables/ fruit containing a large volume of $\mathrm{K}$ is not recommended for patients with severe renal dysfunction because of the risk for hyperkalemia. Ca and $\mathrm{Mg}$ were expected to exhibit hypotensive effects because of the findings of epidemiological studies that blood pressure is low in people who drink hard water, but, in a small-scale interventional study, blood pressure was only slightly decreased with $\mathrm{Ca}$ or $\mathrm{Mg}$ loading. The DASH diet pattern is significant in that even nutrients with mild hypotensive effects may produce a significant hypotensive effect if they are combined.

In Japan, there are few results demonstrating the efficacy of the DASH diet, and recipes for achieving a food composition of a standard Japanese diet corresponding to the DASH diet are not sufficient. However, the 'Japanese Food Guide Spinning Top', which was prepared for healthy people, ${ }^{360}$ may be useful. This guide counts foods according to the DASH diet and recommends 5-6 servings (SVs) of vegetables and $2 \mathrm{SVs}$ of fruit daily. For details, see the reference. ${ }^{360}$ An excessive intake of fruit with a high fructose content is inadvisable for people who need to restrict their energy intake, such as obese and obese patients. The DASH diet has been suggested to have a natriuretic $^{361}$ and a metabolic risk-reducing effect. ${ }^{362}$ As a recent epidemiological study reported that metabolic syndrome was less common in a population with a high $\mathrm{Mg}$ intake, ${ }^{363} \mathrm{Mg}$ may be involved in the latter effect of the DASH diet. The Mediterranean diet is known as a diet resembling the DASH diet. This diet has also been reported to exhibit hypotensive effects and to reduce the risk for cardiovascular disease. 
Table 2 Guidelines for evaluation of salt intake ${ }^{351}$

\begin{tabular}{|c|c|c|}
\hline Primary users & Evaluation method & Recommendability \\
\hline $\begin{array}{l}\text { Special facilities for } \\
\text { hypertension treatment }\end{array}$ & $\begin{array}{l}\text { Measurement of } \mathrm{Na} \text { excretion in } 24-\mathrm{h} \text { pooled urine, weighting } \\
\text { or a } 24-\mathrm{h} \text { recollection dietary survey by a nutritionist. }\end{array}$ & $\begin{array}{l}\text { These methods are highly reliable and recommendable, but are complicated. } \\
\text { Recommended if the cooperation of patients and ability of facilities } \\
\text { are secured. }\end{array}$ \\
\hline $\begin{array}{l}\text { Medical facilities } \\
\text { in general }\end{array}$ & $\begin{array}{l}\text { Measurement of } \mathrm{Na} \text { and } \mathrm{Cr} \text { in second urine after waking } \\
\text { and spot urine, survey regarding the frequency of meals, } \\
\text { dietary history method (estimation using a formula involving } \\
\text { the estimated excretion of } \mathrm{Cr} \text { in } 24-\mathrm{h} \text { urine }^{\mathrm{a}} \text { ). }\end{array}$ & $\begin{array}{l}\text { Although the reliability is relatively low, the method is simple and } \\
\text { is recommended as a practical evaluation procedure. }\end{array}$ \\
\hline Patients themselves & $\begin{array}{l}\text { Estimation in nighttime urine using an electronic salt } \\
\text { sensor installed with a calculation formulab. }\end{array}$ & $\begin{array}{l}\text { Although the reliability is slightly low, the method is recommendable. } \\
\text { It is convenient and can be performed by the patients themselves. }\end{array}$ \\
\hline \multicolumn{3}{|c|}{ 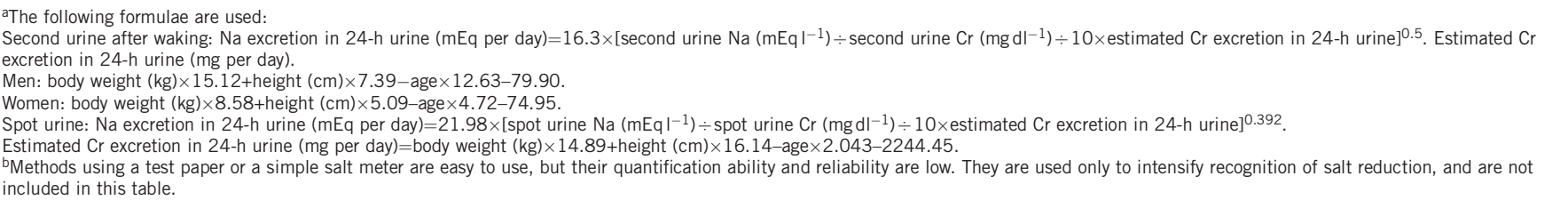 } \\
\hline
\end{tabular}

According to the results of the INTERMAP study, blood pressure tends to be lower in people with a high intake of $n-3$ polyunsaturated fatty acids (rich in fish oil). ${ }^{364} \mathrm{~A}$ meta-analysis of interventional studies showed that an increase in the intake of fish oil caused a decrease in blood pressure in hypertensive patients, although a relatively high dose ( $3 \mathrm{~g}$ per day or more) was necessary to achieve a significant hypotensive effect. ${ }^{365}$ Incidentally, the 'Japanese Food Guide Spinning Top' ${ }^{360}$ recommends two SVs of fish daily. Moreover, a cohort study in Japan (Japan Public Health Center-Based [JPHC] Study) reported a lower incidence of myocardial infarction in people with a higher fish intake. ${ }^{366}$ On the other hand, in a recent interventional study regarding n-3 fatty acid (mainly eicosapentaenoic acid and docosahexaenoic acid) intake, there was no decrease in the risk for cardiovascular disease. ${ }^{367}$ In Japan, a study indicated that a high-purity eicosapentaenoic acid preparation decreased the risk for coronary artery disease. ${ }^{368}$ In addition to the type/dose, this issue must be further examined, considering regional differences (fish intake is essentially high in the Japanese).

According to the OmniHeart Study involving hypertensive patients, ${ }^{369}$ a protein- or unsaturated fatty acid-rich diet was more useful than a carbohydrate-rich diet for decreasing blood pressure and reducing the metabolic risk. The usefulness of unsaturated fatty acids is compatible with the results of the DASH diet. ${ }^{288,339}$ The significance of dietary patterns including an increased intake of protein and carbohydrate restriction must be further investigated in the future. A meta-analysis demonstrated the hypotensive effects of dietary fiber. ${ }^{370}$ Regarding the effects of an increased intake of antioxidant foods on blood pressure, there is no evidence worth mentioning in the guidelines for the management of hypertension.

\section{MAINTAINING A PROPER BODY WEIGHT}

As obesity is an important etiological factor for hypertension, obese individuals should reduce their body mass index ([body weight $(\mathrm{kg})] \div[\text { height }(\mathrm{m})]^{2}$ ) to $<25 \mathrm{~kg} \mathrm{~m}^{-2}$, and non-obese individuals should maintain this level. Obesity induces not only hypertension but also abnormalities of glucose/lipid/uric acid metabolism, cerebral infarction, fatty liver, menstrual disorder, pregnancy complications, sleep apnea syndrome, obesity hypoventilation syndrome, orthopedic diseases and obesity-associated nephropathy. ${ }^{371}$ Obesity-associated nephropathy ${ }^{372}$ has recently been emphasized. Obesity, especially severe obesity, causes proteinuria and subsequently kidney dysfunction. Furthermore, obesity is also a factor involved in the deterioration of chronic kidney disease. Kidney dysfunction makes hypertension severer, and weight control is important in hypertensive patients. In individuals with a higher visceral fat level, the incidences of hypertension, dyslipidemia and hyperglycemia are higher. ${ }^{373}$ Therefore, waist circumference should also be considered when practicing weight control ( $<85 \mathrm{~cm}$ in men, $<90 \mathrm{~cm}$ in women). ${ }^{371}$

The hypotensive effect of weight loss has been established. According to a recent meta-analysis, blood pressure was significantly $(-4.5 /$ $-3.2 \mathrm{~mm} \mathrm{Hg}$ ) decreased by a weight loss of $\sim 4 \mathrm{~kg} .{ }^{374}$ Obese hypertensive patients should first practice weight control, but should follow this with a stress-free, long-term weight-loss plan, considering that rapid weight loss may induce adverse events and that a significant decrease in blood pressure is achieved by a weight loss of $\sim 4 \mathrm{~kg}$. Childhood obesity is an important etiological factor for hypertension. In particular, adequate guidance/education for the prevention of obesity should be performed in children.

\section{EXERCISE}

The hypotensive effect of aerobic exercise has been established. ${ }^{342}$ Increased physical activities have been shown to reduce blood pressure, decrease body weight, body fat and waist circumference, and improve insulin sensitivity and serum lipid levels. In addition, a low physical activity level increases the risk of cardiovascular disease. ${ }^{375}$ An exercise therapy-related increase in oxygen intake may contribute to an improvement in prognosis. Therefore, exercise is recommended for hypertensive patients as a lifestyle modification.

Aerobic exercise such as fast walking is suitable for the prevention and treatment of lifestyle-related diseases, including hypertension. Concerning the intensity of exercise, assessment scales vary among reports. Although the evidence level is not high, many guidelines recommend that the intensity of exercise should be established as $50 \%$ of maximum oxygen intake. This corresponds to 'slightly hard' in the Borg scale, in which the intensity of exercise is estimated on the basis of subjective findings. However, in the American College of Sports Medicine/AHA recommendations for the general public, it is described that mixing high- with moderate-intensity exercise is more useful for reducing the risk of cardiovascular disease. ${ }^{376}$ In hypertensive patients, however, blood pressure markedly increases during exercise if intensity is too high, ${ }^{377}$ and high-intensity exercise has been reported to exacerbate the prognosis, unlike that 
in normotensive individuals. ${ }^{378}$ Therefore, strenuous exercise should be carefully conducted in hypertensive patients. Exercise should be performed periodically - that is, for $30 \mathrm{~min}$ or more, daily, if possible. ${ }^{379}$ In the American College of Sports Medicine/AHA recommendations for the general public, ${ }^{376}$ it is described that patients may be regarded as having achieved this goal if exercise of at least 10-min duration has been repeated to achieve a daily total of $\geqslant 30 \mathrm{~min}$. If resistance and stretching exercise is combined with aerobic exercise, the former is useful for increasing lean body mass, preventing osteoporosis and lowering back pain, and the latter improves the motion range and function of joints. Recently, a meta-analysis showed that resistance exercise exhibited hypotensive effects. ${ }^{380}$ 'The Exercise Guide $2006^{381}$ classified physical activities into exercise and activities of daily living, and proposed an approach to increase the physical activity level primarily by increasing the latter. In patient education also, physicians should instruct patients to increase the physical activity level of their daily lives (for details, see the reference).

Candidates for exercise therapy are grade II or lower hypertensive patients with no cardiovascular disease (in patients with grade III or higher hypertension, exercise therapy is performed after a decrease in blood pressure is achieved). In high-risk patients, a medical check must be performed in advance, and exercise should be restricted or provided if necessary. Exercise should not be restricted owing to old age alone, but a prior medical check is particularly necessary in elderly persons.

\section{REDUCTION OF ALCOHOL INTAKE}

Habitual alcohol consumption can lead to an increase in blood pressure. ${ }^{382,383}$ Heavy drinking induces hypertension, and can also cause stroke, alcoholic cardiomyopathy, atrial fibrillation and nocturnal sleep apnea, as well as cancer, increasing the mortality rate. An epidemiological study indicated that moderate drinking reduced the risk of cardiovascular disease in hypertensive patients and that there was a U-shaped relationship between the volume of alcohol and risk for cardiovascular disease. ${ }^{384}$ However, the protective effect of moderate drinking on the cardiovascular system, including its mechanism, must be examined in the future.

A bolus administration of alcohol causes a decrease in blood pressure that is sustained for several hours, ${ }^{385}$ but if it is continued for a long period blood pressure increases. It has been reported that about an $80 \%$ reduction in alcohol intake is followed by a decrease in blood pressure in 1-2 weeks. ${ }^{386}$ A meta-analysis also showed the hypotensive effects of alcohol restriction. ${ }^{342}$ In heavy drinkers, blood pressure is increased after an abrupt reduction of drinking, but it can be reduced if the restriction is continued. Drinking, in terms of ethanol intake, should be restricted to $20-30 \mathrm{ml}$ (equivalent to $180 \mathrm{ml}$ of sake, $500 \mathrm{ml}$ of beer, $<90 \mathrm{ml}$ of shochu, a double whisky or brandy and 2 glasses of wine)/day or less in $\mathrm{men}^{387}$ and to $10-20 \mathrm{ml}$ per day or less in women.

\section{SMOKING CESSATION}

Despite recent reports on the effect of smoking on the development of hypertension, ${ }^{388}$ the overall effect of smoking on blood pressure has not been established. However, smoking a cigarette causes an increase in blood pressure persisting for $15 \mathrm{~min}$ or more. ${ }^{389}$ Therefore, smokers frequently develop masked hypertension with an increase in daytime ambulatory blood pressure. ${ }^{390}$ Furthermore, smoking does not cause an increase in brachial blood pressure, but increases the amplification index or central blood pressure. ${ }^{391}$ If smoking cessation is successful, these values decrease. ${ }^{392}$ Smoking is known to be a risk factor for renovascular hypertension. ${ }^{393}$ On the other hand, a study reported that smoking cessation led to a 2-kg weight gain and an increase in blood pressure, although it decreased the risk for coronary artery disease. $^{394}$ Weight gain related to dietary changes after smoking cessation must be controlled.

Smoking is a strong risk factor not only for noncardiovascular diseases including cancer but also for coronary artery disease and stroke. Considering that the purpose of hypertension treatment is to prevent cardiovascular disease, smoking cessation is important regardless of the influence on blood pressure. Non-smokers around smokers are also affected, and even passive smoking increases these risks. It is reported that, in passive smokers, 24-h blood pressure is high, and the incidence of masked hypertension is also high. ${ }^{395}$ Not only hypertensive patients but also healthy people should quit smoking. For guidance regarding smoking cessation, the 'Guidelines for Smoking Cessation'396 and 'Standard Procedures for Smoking Cessation Treatment, ${ }^{397}$ prepared by a collaborative study group consisting of many academic societies, are useful. Smoking cessation-assisting drugs (varenicline, nicotine replacement therapy) should also be considered if necessary. If patients meet specific conditions, therapy for smoking cessation is covered by health insurance in Japan.

\section{OTHER LIFESTYLE MODIFICATIONS}

Exposure to cold elevates blood pressure, which, consequently, is increased during winter. The cardiovascular mortality rate during winter is greater when protective measures against the cold are inadequate. ${ }^{398}$ Therefore, the homes of hypertensive patients should be adequately heated in winter, with particular attention to heating of the toilet, bathroom and dressing room, which is often disregarded.

Reports on the relationship between emotional stress and blood pressure are contradictory, but a recent meta-analysis indicated the effectiveness of stress management. ${ }^{342}$ Therefore, stress-controlling techniques such as biofeedback may be worth attempting in some hypertensive patients. ${ }^{379}$

Epidemiological studies have indicated the possibility that the duration and quality of sleep may be related to an increase in blood pressure and an increase in the risk for cardiovascular disease. ${ }^{399-401}$ In some patients, sleep improvement may be useful for decreasing blood pressure.

When bathing, the water should not be too hot. Blood pressure has been reported to show little increase when bathroom temperature is $\geqslant 20{ }^{\circ} \mathrm{C}$ and water temperature is $\leqslant 40{ }^{\circ} \mathrm{C}$. A water temperature of $38-42^{\circ} \mathrm{C}$ and a duration of $5-1 \mathrm{~min}$ are advisable when taking a bath. The water temperature of sento (Japanese bathhouses) is often too high. Hypertensive patients should avoid bathing in cold water and using saunas.

As straining to defecate increases the blood pressure, guidance for the prevention of constipation should be given, and, if necessary, laxatives should be administered.

Sexual intercourse also raises the blood pressure, but hypertension poses few problems to one's sex life. However, hypertensive patients with cardiovascular diseases should refrain from vigorous sexual activity.

\section{COMBINED LIFESTYLE MODIFICATIONS}

The DASH ${ }^{288}$ and DASH-Sodium ${ }^{339}$ studies suggested that combined improvements in diet facilitate a marked decrease in blood pressure. Also, the TONE study ${ }^{343}$ showed that a combination of salt reduction and weight loss is more likely to reduce blood pressure and prevent cardiovascular diseases even when they are practiced less rigorously. A more marked decrease in blood pressure has been reported to be achieved by a combination of salt intake reduction, weight loss, 
exercise, restriction of alcohol intake and a DASH diet. ${ }^{271}$ Therefore, combined lifestyle modifications are recommended. Lifestyle modifications should be started in childhood to prevent lifestyle-related diseases, including hypertension.

\section{FOODS FOR SPECIFIED HEALTH USES (FOSHUS)}

FOSHU refers to foods to be ingested for specified health purposes, permitted according to Clause 1, Article 26, of the Health Promotion Act or approved according to Clause 1, Article 29, of the same act (Commissioner of the Consumer Affairs Agency), the packages containing which describe that food consumption may achieve the health purpose. Food with health function that is not substantiated by scientific evidence that meets the level of FOSHU, or food with certain effectiveness but without the established mechanism of the effective element for the function, will be approved as qualified FOSHU. They are expressed with marks shown in Figure 4-2. The hypotensive effects of foods considered effective for blood pressure control are often based on an ACE-inhibiting activity, but the indicated 'recommended daily intake' should be strictly observed when consuming these foods. In addition, physicians must instruct pregnant women and patients with nephropathy to pay attention to the recommended daily intake. Patients must also be informed that the intake of FOSHU cannot be a substitute for antihypertensive medication. A warning to consult a physician should be given to patients already on antihypertensive medication if they wish to use such foods. Information on FOSHU is available on the homepage
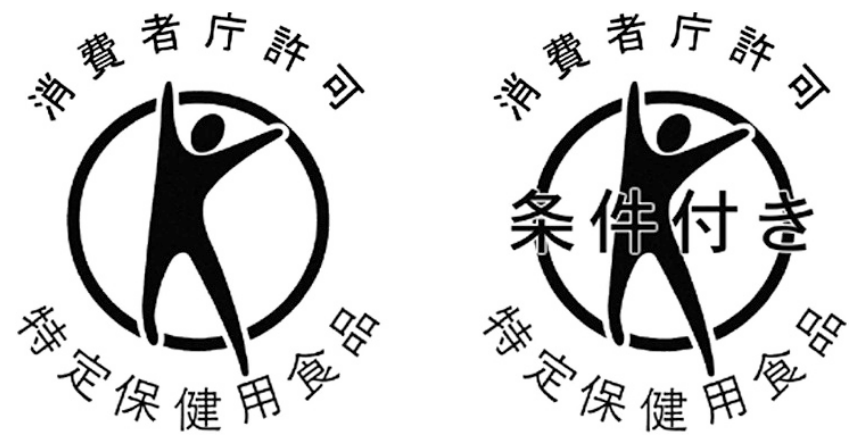

Figure 4-2 Marks of foods for specified health uses.

of the Consumer Affairs Agency (http://www.caa.go.jp/foods/ index4.html) or in the National Institute of Health and Nutrition (http://hfnet.nih.go.jp/contents/detail773.html).

\section{Citation Information}

We recommend that any citations to information in the Guidelines are presented in the following format:

The Japanese Society of Hypertension Guidelines for the Management of Hypertension (JSH 2014). Hypertens Res 2014; 37: 253-392.

Please refer to the title page for the full list of authors. 